\title{
Enterolitíase em equinos da raça crioula
}

\author{
Enterolithiasis in Crioulo Horses \\ Alice Correa Santos' ${ }^{1}$ Bruna da Rosa Curcio² ${ }^{2}$ llusca Sampaio Finger ${ }^{3}$, \\ Jarbas Castro Junior 4 Carlos Eduardo Wayne Nogueira ${ }^{2}$
}

\begin{abstract}
Background: Enteroliths are intestinal mineral calculi predominantly composed of struvite. In horses this material accumulates concentrically around a core, causing total or partial obstruction of the higher and lower colon, and can lead to death by rupture. The enterolithiasis has worldwide distribution, and occurs frequently in Rio Grande do Sul, especially in Crioulo Horses. The aim of this work is to report a sequence of cases of Crioulo Horses with enterolithiasis, linking risk factors through historical analysis, clinical presentation and management employed by the breeding farms.

Case: Between the years 2012 and 2015, 16 Crioulo Horses with colic syndrome caused by enteroliths were treated at the Hospital de Clínicas Veterinária of Universidade Federal de Pelotas (UFPel), RS, Brazil and Clínica Hípica, in Porto Alegre, RS. The group was formed predominantly by males, weighted $430 \mathrm{~kg}$ in average and the average age was eight years old. All horses underwent general and specific clinical examination, laboratory tests and surgical procedure to remove the enterolith. After discharge, there was an epidemiological questionnaire seeking information about the history, food and environmental management that could be related to the development of enterolithiasis. In relationship to the history of previous episodes of colic, the results were: 4 horses (25\%) had no previous episodes of colic, while 12 horses (75\%) had experienced previous colic. Regarding the horses' bedding, 70\% use sawdust and 30\% rice husk, and about behavior, ten $(62.5 \%)$ of the 16 horses attended had no stereotypies. Related to food and water supply, it was observed that nine horses $(56.25 \%)$ had alfalfa as the only roughage source in the diet, and all animals (100\%) were fed commercial food and water ad libitum. According to the number of hours the patients remained stabled, the rearing system was classified as intensive (over $12 \mathrm{~h} /$ stable/day), extensive ( $0 \mathrm{~h}$, at the field) or semi-extensive (up to $12 \mathrm{~h} /$ stable/day). The frequencies observed were: intensive ( 9 animals - 56.25\%), semi-intensive ( 6 animals - 37.5\%) and extensive ( 1 animal - 6.25\%). The average time of stabling was $17.2 \mathrm{~h} /$ day.

Discussion: The alfalfa consumption as the only forage observed in our study is the main risk factor described for the formation of enteroliths. This is because in digestion, alfalfa tends to alkalize the intestinal $\mathrm{pH}$, hindering the metabolism of some minerals and thus forming the enterolith. Another important risk factor observed in this work is the intensive rearing, with long periods of stabling. This explains why confinement leads to decreased intestinal motility, due to reduced or absent grazing. Furthermore, reduced physical activity is another contributing factor to the reduction of motility, because the exercise influence the intestinal peristalsis. The rearing of horses intensively is usual with the Crioulo, especially in training and morphological preparation. Based on this case study, we can't say that there is a racial predisposition to enterolithiasis of the Crioulo Horse, since it would require more studies on the physiology and a standard feeding of these horses. However, we can attribute the considerable volume of enteroliths observed in Crioulo Horses in Rio Grande do Sul to the increase in the number of referrals to the reference hospitals, feeding alfalfa as the only roughage source in the diet and to intensive management of the farms, with long periods of daily stabling.
\end{abstract}

Keywords: enteroliths, horses, Crioulo Horse.

Descritores: enterólitos, cavalos, raça Crioula. 


\section{INTRODUÇÃO}

A síndrome cólica em equinos é caracterizada por dor abdominal na maioria dos casos [3]. Os motivos da predisposição dos equinos ao desenvolvimento de cólicas são diversos, incluindo mudanças alimentares, dietas ricas em concentrado, restrição na ingesta de água, controle parasitário deficiente e fatores relacionados ao manejo [11].

As cólicas obstrutivas primárias representam grande incidência em animais atendidos em hospitais de referência. Os enterólitos são cálculos minerais que em eqüinos são compostos predominantemente de estruvita (fosfato de amônio e magnésio) [7,13], encontrados em dietas ricas em magnésio, nitrogênio e fósforo. Este material acumula-se concentricamente ao redor de um núcleo, causando obstruções totais ou parciais no cólon maior e cólon menor, gerando quadros de dor acentuada durante a movimentação no lúmen intestinal, que pode evoluir com a ruptura de alças intestinais e morte [7].

A ocorrência de casos de enterolitíase tem distribuição mundial, e embora contraste com a opinião de outros autores, Hassel et al. [7] afirmam que não há predisposição racial envolvida. Contudo, têm sido cada vez mais observados casos em hospitais e clínicas veterinárias no Rio Grande do Sul [10], predominantemente na raça Crioula.

O objetivo deste trabalho é relatar uma série de casos de enterolitíase em equinos da raça Crioula, relacionando fatores de risco através da análise do histórico, apresentação clínica e manejo empregado nos criatórios da raça.

\section{CASOS}

Entre os anos de 2012 e 2015, foram atendidos 16 animais da raça Crioula com síndrome cólica causada por enterólitos. Estes foram encaminhados ao Hospital de Clínicas Veterinária da Universidade Federal de Pelotas (RS) e Clínica Hípica, em Porto Alegre (RS) com sintomatologia de dor abdominal intensa. A média de peso dos animais era de $430 \mathrm{~kg}$, com média de idade de 8 anos (3 - 16 anos e Desvio Padrão: 3,18), sendo onze machos e cinco fêmeas.

Todos equinos foram submetidos a exame clínico geral e específico do sistema gastrointestinal. Foram realizados exames complementares, como hemograma, sondagem nasogástrica, palpação transretal e paracentese abdominal, a fim de estabelecer a conduta clínica determinando a necessidade do tratamento cirúrgico.

O procedimento cirúrgico consistiu de laparatomia exploratória sob anestesia geral inalatória, com o objetivo de localizar e remover o enterólito (Figura 1). Doze animais $(75 \%)$ apresentaram apenas um enterólito, enquanto quatro (25\%) apresentaram dois. Destes, treze $(65 \%)$ localizados no cólon maior e sete (35\%) no cólon menor. Quanto ao formato, quatorze $(87,5 \%)$ apresentaram formato arredondado, e dois $(12,5 \%)$, formato irregular.

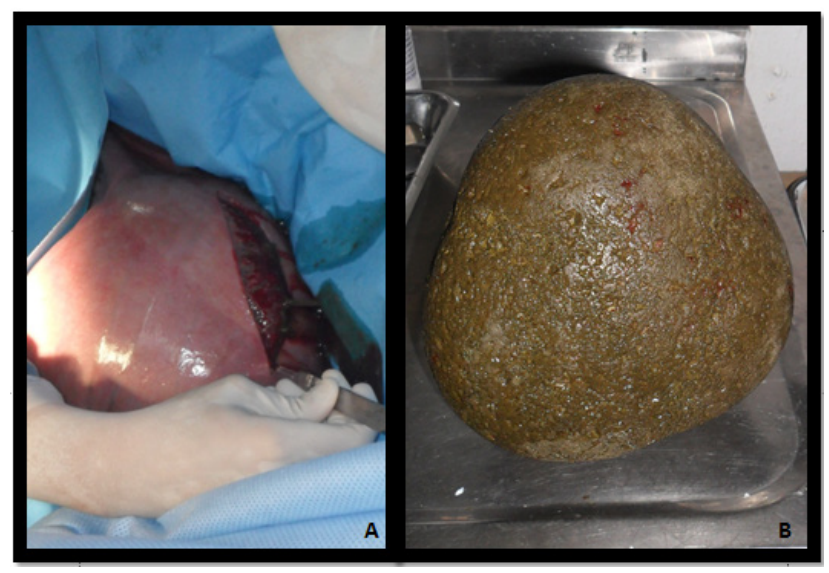

Figura 1. Procedimento de laparatomia exploratória para retirada de enterólito de um dos pacientes estudados. A) Localização e retirada do enterólito no cólon maior. B) Enterólito após a remoção, peso 4.200Kg.

Após a alta médica, realizou-se um questionário epidemiológico buscando informações acerca do histórico, manejo alimentar e ambiental que pudessem estar relacionadas ao desenvolvimento de enterolitíase.

Quando questionados a respeito do histórico de episódios anteriores de cólica, os resultados foram: 4 equinos (25\%) não apresentaram episódios anteriores de cólica, enquanto 12 equinos $(75 \%)$ apresentaram sinais de cólica anteriormente. No histórico de afecções dentárias, nove $(56,25 \%)$ animais apresentaram e sete $(43,75 \%)$ nunca tiveram problemas dentários ou não realizaram avaliação odontológica anterior.

Com relação à cama das cocheiras, $70 \%$ utilizavam serragem e $30 \%$ casca de arroz, e dez (62,5\%) dos 16 equinos atendidos não apresentava estereotipias. Quando questionados a respeito da alimentação e oferta de água, observou-se que nove equinos $(56,25 \%)$ tinham a alfafa como único volumoso na alimentação, e todos os animais (100\%) recebiam ração comercial e água ad libitum. 
Com base no número de horas que os pacientes permaneciam estabulados, foram classificados de acordo com o sistema de criação em intensivo (acima de $12 \mathrm{~h} /$ cocheira/dia), extensivo ( $0 \mathrm{~h}$, a campo) ou semi-extensivo (até $12 \mathrm{~h} /$ cocheira/dia). As frequências observadas foram: intensivo (9 animais - 56,25\%), semi-intensivo (6 animais - 37,5\%) e extensivo (1 animal $-6,25 \%)$. A média de tempo de estabulação era de 17,2 h/dia, independente do sistema de criação.

\section{DISCUSSÃO}

A média de idade para ocorrência de enterolitíase é de 8 a 12 anos [1], semelhante ao encontrado nos animais avaliados, que tiveram média de 8 anos de idade. Embora a ocorrência em animais menores de quatro anos seja rara [1], em nosso estudo observamos uma distribuição heterogênea de idade nos animais acometidos, variando de três a dezesseis anos. De acordo com os demais dados colhidos em nosso estudo, podemos considerar que a ocorrência esteja muito mais relacionada ao tempo e intensidade de exposição aos fatores de risco, como estabulação e alimentação incorreta, do que à idade propriamente dita.

Houve maior ocorrência em machos quando comparado às fêmeas, embora a enterolitíase não pareça ter relação com o gênero [5]. É possível que a maior ocorrência nos machos esteja relacionada com o manejo intensivo ao qual estão condicionados (especialmente garanhões). Apesar da evidência, em nosso estudo de casos, trabalhamos com um grupo heterogêneo (68,75\% machos e 31,25\% fêmeas), e o maior número de machos não permite afirmar predisposição do gênero.

Nos animais estudados predominaram enterólitos únicos, que também foram os mais comumente observados por outros autores [6,8], embora não seja rara a ocorrência de vários enterólitos em um mesmo animal. O formato arredondado ocorreu em 87,5\% dos casos acompanhados, que segundo Hassel et al. $[6,8]$ está relacionado com a deposição concêntrica de minerais, decorrente do tempo de formação e desgaste da superfície através da motilidade intestinal.

A porção intestinal que apresentou maior ocorrência de enterólitos foi o cólon maior (65\%), embora possam ser formados também no cólon menor [8]. Como a motilidade intestinal promove a movimentação dos cálculos no lúmen intestinal [13], podemos sugerir que o local de obstrução localizado na laparatomia exploratória não necessariamente seja o local de formação do enterólito.

A cólica causada por enterólitos geralmente cursa com quadros de dor abdominal intermitentes, sendo essa a apresentação clássica $[6,7,13]$. Corroborando com estes, em nosso trabalho, a maioria dos animais relatados $(75 \%)$ apresentaram episódios de cólicas anteriores.

Dos animais atendidos, 56,25\% tinham a alimentação volumosa exclusivamente de feno de alfafa. O consumo deste volumoso é descrito como um dos principais fatores de risco para a formação de enterólitos [1,6,13]. A alfafa, por ser altamente proteica, ao ser digerida resulta em amônia, que alcaliniza o $\mathrm{pH}$ intestinal. Em razão da elevação do $\mathrm{pH}$, alguns minerais não são digeridos, levando à sua deposição ao redor de um núcleo, favorecendo a formação do enterólito [6].

Todos os animais recebiam ração comercial. Com base em nosso estudo, não é possível atribuir relação entre a ingestão de ração e a formação de enterólitos, por não termos parâmetro comparativo com outros tipos de concentrado. Entretanto, é importante salientar que embora as rações comerciais no Brasil atendam aos valores mínimos exigidos de cálcio, magnésio e fósforo pelo National Research Council (NRC) [12], os valores máximos não são conhecidos, e esses são os minerais mais facilmente encontrados em excesso no trato intestinal de equinos com enterolitíase [8].

Embora em nosso estudo a cama de serragem tenha sido predominantemente utilizada para os equinos com enterolitíase, outros autores citam que não há relação entre o material da cama e a formação de enterólitos [8]. Mais relevante que isto são as alterações comportamentais que podem levar a ingestão de cama, bem como as demais estereotipias. Neste contexto, observamos que a maioria dos animais não apresentou estereotipias $(62,5 \%)$, sugerindo que essas não tenham relação direta com a formação de enterólitos nos cavalos da raça Crioula que estudamos. Entretanto, em estudos anteriores, animais da raça Crioula em regime predominantemente intensivo apresentaram maior predisposição ao desenvolvimento de estereotipias [2], que estão diretamente ligadas ao estresse, podendo causar afecções estomacais e cólicas [15]. Na raça Percheron e Bretã foi observado que animais em regime de confinamento passaram $89 \%$ do tempo ociosos na baia, favorecendo o desenvolvimento de distúrbios comportamentais [14]. Estes dados de- 
monstram que, embora em nosso trabalho não esteja diretamente relacionado à ocorrência de enterólitos, o manejo intensivo é extremamente danoso para a saúde física e mental do cavalo.

O tempo de estabulação diário e o manejo intensivo ao qual estavam condicionados a maior parte dos pacientes estudados é um dado que associamos a ocorrência de enterolitíase. O confinamento por longos períodos está relacionado com a diminuição da motilidade intestinal, através do pastejo reduzido ou ausente, levando a uma predisposição para formar enterólitos [7,13]. Além disso, o curto período de atividade física é outro fator agravante para a redução da motilidade, já que o exercício influencia no peristaltismo intestinal [7].

A manutenção de equinos confinados para treinamento e preparo morfológico é frequente em animais da raça Crioula [4]. Não é possível afirmar que haja uma predisposição racial, mas certamente o manejo intensivo tem íntima relação com o elevado número de cólicas por enterolitíase que se observa em animais desta raça. História semelhante foi observada na raça Árabe, descrita como predisposta a enterolitíase por Lloyd et al. [9]. Bray [1] contrapôs essa afirmação, citando que essa predisposição não possui embasamento fisiológico, já que na época a alimentação de equinos
Árabes consistia em grãos e feno de alfafa. Além disso, a raça estava em alta no mercado e os proprietários dispostos a investir em procedimentos cirúrgicos, o que elevava o número de diagnósticos.

$\mathrm{Na}$ raça Crioula observa-se um cenário econômico de semelhante expansão, onde a modalidade de provas funcionais apresenta o maior número de animais em atividade [4] e há um maior investimento financeiro na saúde dos animais. Somando essa questão econômica com a alimentação rica em alfafa, temos atualmente nos equinos Crioulos uma situação semelhante à descrita naquela época nos cavalos Árabes [1]

Com base nesse estudo de casos, não podemos afirmar que exista uma predisposição racial para ocorrência de enterolitíase em equinos da raça Crioula, pois seriam necessários mais estudos acerca da fisiologia e padrão alimentar desses animais. Contudo, podemos atribuir o número de equinos da raça Crioula com enterólitos no Rio Grande do Sul ao manejo intensivo dos criatórios com alimentação de alfafa e longos períodos de estabulação diários, além da possibilidade de diagnostico através da laparatomia exploratória, realizada nos hospitais de referencia do Estado.

Declaration of interest. The authors report no conflicts of interest. The authors alone are responsible for the content and writing of the paper.

\section{REFERENCES}

1 Bray E.B. 1995. Enteroliths: feeding and management recommendations. Journal of Equine Veterinary Science. 15(11): 474-478.

2 Canal Jr. A. 2015. Influência do tempo de estabulação no comportamento de equinos da raça Crioula. Unoesc \& Ciência. 6(2): 203-2010.

3 Cook L.V. \& Hassel D.M. 2014. Evaluation of the colic in horses: decision for referral. Veterinary Clinics of North America: Equine Practice. 30: 383-388.

4 Gallio M., Azevedo M.S., Brass K.E., De La Corte F.D. \& Lopes L.F.D. 2014. Prevalência de alterações ósseas no tarso de potros Crioulos de até vinte e seis meses de idade. Ciência Rural. 44 (8): 1442-1447.

5 Hassel D.M., Snyder J.R., Langer D.M., Drake C.M. \& Yarbrough T.B. 1997. Equine enterolithiasis: a review and retrospective analysis of 900 Cases (1973-1996). In: Proceedings of the Annual Convention of the AAEP (Phoenix, EUA). p.246.

6 Hassel D. 2002. Enterolithiasis. Clinical Techniques in Equine Practice. 1(3): 143-147.

7 Hassel D.M., Aldridge B.M., Drake C.M. \& Snyder J.R. 2008. Evaluation of dietary and management risk factors for enterolithiasis among horses in California. Research in Veterinary Science. 85: 476-480.

8 Hassel D.M., Spier S.J., Aldridge B.M., Watnick M., Argenzio R.A. \& Snyder J.R. 2009. Influence of diet and water supply on mineral content and $\mathrm{pH}$ within the large intestine of horses with enterolithiasis. The Veterinary Journal. 182: 44-49.

9 Lloyd K., Hintz H.F., Wheat J.D. \& Schryver H.F. 1987. Enteroliths in horses. Cornell Veterinarian. 77: $172-186$.

10 Markus D., Lins L.A., Vieira J., Castro Jr. J. \& Nogueira C.E.W.N. 2007. Casos de cólica em equinos com necessidade de intervenção cirúrgica, em dois centros cirúrgicos na cidade de Porto Alegre, durante o primeiro semestre de 2006. In: XVI Congresso de Iniciação Científica da Universidade Federal de Pelotas (Pelotas, Brasil). 1 CD ROM. 
11 Mehdi S. \& Mohammad V. 2006. A farm-based prospective study of equine colic incidence and associated risk factors. Journal of Equine Veterinary Science. 26(4): 171-174.

12 National Research Council - NRC. 2007. Nutrient requirements of horses. 6.ed. Washington, D.C.: National Academies Press, 341p.

13 Pierce R. P. 2009. Enteroliths and other foreign bodies. Veterinary Clinics of North America: Equine Practice. 25: 329-340. 14 Rezende M. J. M., Pimentel C.M.M., Martins R. D., Oliveira L.P.G., Garcia J.A.S. \& Louvandini H. 2006. Comportamento de cavalos estabulados do exército brasileiro em Brasília. Ciência Animal Brasileira. 7(3): $327-337$.

15 Wickens C.L. \& Heleski C.R. 2010. Crib-biting behavior in horses: a review. Applied Animal Behavior Science. 128:1-9. 